ORIGINAL ARTICLE

\title{
Neonatal weight loss in breast and formula fed infants
}

\author{
P D Macdonald, S R M Ross, L Grant, D Young
}

Arch Dis Child Fetal Neonatal Ed 2003;88:F472-F476

See end of article for authors' affiliations

.....................

Correspondence to: Dr Macdonald, Neonatal Paediatric Department, Southern General Hospital, Glasgow G51 4TF, Scotland, UK; peter.macdonald@ sgh.scot.nhs.uk

Accepted 17 December 2002

\begin{abstract}
Objective: To define the range of neonatal weight loss in a population relative to feeding method. Design: Prospective observational cohort study. Setting: Maternity service providing geographically defined, community based newborn follow up. Participants: 971 consecutive term newborns of birth weight $\geqslant 2500 \mathrm{~g}$ during the first 2-3 weeks of life; 34 excluded (inadequate data). 937 included: $45 \%$ breast fed, $42 \%$ formula fed, 13\% breast and formula fed.

Outcome measures: Maximum weight loss and timing, age on regaining birth weight.

Results: Median weight loss: formula fed 3.5\%, breast fed 6.6\%. Upper centiles for maximum weight loss differ considerably (95th centiles: breast fed $=11.8 \%$, formula fed $=8.4 \%$; 97.5 th centiles: breast fed $=12.8 \%$, formula fed $=9.5 \%$ ). Median time of maximum weight loss: 2.7 days for breast fed and formula fed. Recovery of birth weight: breast fed median 8.3 days, 95th centile 18.7 days, 97.5 th centile 21.0 days; formula fed median 6.5 days, 95th centile 14.5 days, 97.5 th centile 16.7 days. The time taken to regain birth weight correlates with both the degree and timing of initial weight loss for all groups. Conclusions: Early neonatal weight loss is defined allowing identification of infants who merit closer assessment and support.
\end{abstract}

W e have observed an increase in the number of breast fed babies presenting with dehydration and/or failure to thrive because of lactation failure and non-recognition of feeding problems. Recent reports ${ }^{12}$ support this experience and recommend monitoring of the weight of infants through the neonatal period. However, these reports acknowledge uncertainty as to what actually constitutes normal neonatal weight loss. Maisels and colleagues published two studies which have been quoted as giving guidance on normal loss. Both studies were designed primarily to study factors that influence breast milk jaundice. The first ${ }^{3}$ reported a mean weight loss of about $6 \%$ in 100 unselected well babies during the first 3 days. The subsequent study ${ }^{4}$ reported a mean weight loss of $6.86 \%$ in 186 infants. The timescale over which babies were weighed was not clearly indicated, although it may have only been 23 days. The sample was neither population based nor randomly selected, being largely preselected because of the presence of more pronounced jaundice. The distribution of data points for early neonatal weight loss are likely to be skewed, yet both studies reported the results as mean (SD). Owing to the design and method of data presentation, these studies cannot reliably inform the debate as to what constitutes the norm. Marchini and colleagues published reports also designed primarily to study other issues. One ${ }^{5}$ indicated a mean early weight loss of $5.7 \%$. Measurements were recorded over a three day period, and no indication is given of the skewness of the data. Another study ${ }^{6}$ reported a median weight loss of about $6 \%$ recorded over a four day period. At least one baby lost $>15 \%$ of his/her birth weight during this time, but there is no clear information as to the frequency with which more extreme degrees of weight loss are observed.

These studies give limited guidance on population averages, but cannot provide robust data on extremes of weight change within a population of newborns.

Local clinical guidelines stipulate that all babies followed up by our community midwives should be weighed regularly during the first two weeks. Recognising the weakness of the current information on normal weight loss, we reviewed the collected data, with a view to answering three questions.

- What percentage of their birth weight do babies initially lose?

- How long does it take for babies to reach their weight nadir?

- How long does it then take to regain the birth weight?

We sought to identify upper limits to these parameters so as to provide a sound evidence base for the identification of high risk infants.

\section{METHODS}

Our midwives are responsible for home follow up of a geographically defined population of newborns based on local postcode. In hospital, babies were weighed at birth and before discharge (around 48 hours). Further weights were recorded at home on about the 5th, 7th, and 10th days of life. Weighing was discontinued once an infant had regained his/ her birth weight. As this took a variable length of time, some infants were followed up for longer and had more weights recorded than others. Those babies not regaining their birth weight by day 10 were reweighed at 2 weeks of age. The guidelines were designed for practical use, and these times were not prescriptive. Midwives recorded the date and time of each weight to allow calculation of precise age. Infants were weighed naked using a set of digital scales, and the weight was expressed in kilograms. Each team of midwives was allocated a single set of scales and had responsibility for specific individual patients. The scales were purchased just before the start of the project; they were calibrated before initial use and are recalibrated by the manufacturer at least every six months.

To exclude any underlying condition and to check for dehydration, babies who lost more than $10 \%$ of their birth weight were referred for medical review at their hospital of birth. Estimation of the plasma electrolytes was used in some infants (those born in our hospital) as part of this 
assessment. Those babies not regaining their birth weight by day 14 of life also received further follow up. This involved maintaining midwifery input to support feeding and offering review appointments in the infant follow up clinic.

We reviewed the data collected between March and July 2000 on all babies $\geqslant 2500 \mathrm{~g}$ and $\geqslant 37$ weeks gestation. Of 971 such babies, virtually all had two or three follow up weights, more than half the infants had four follow up weights, over a quarter of the breast fed infants had five or more weights (some having seven or eight); see table 1 for numbers and timing. Thirty four infants were not weighed sufficiently often to record a minimum weight (usually only having one postnatal weight) and were excluded. Included in the analysis were 937 babies: 420 were exclusively breast fed, 396 were exclusively formula fed, and 121 babies were "mixed fed" either switching to formula feeding from breast feeding within the time period or having supplementary feeds from the start. To standardise the measurements, we expressed each infant's recorded weights as a ratio over birth weight. We took the lowest recorded ratio and its timing as an approximation for the degree and timing of the individual's maximum weight loss. On the occasions (4\%) when there were two identical lowest weight records, we took the timing of the weight nadir to be the midpoint of the two records.

We estimated the time taken to regain birth weight by one of two methods. For most infants, we assumed linear weight gain between the last recording with a current weight/birth weight ratio $<1.0$ and the first recording at or above the birth weight. A total of 118 infants ( 66 breast fed, 38 formula fed, 14 mixed fed) did not have a weight actually measured at or above their birth weight. If these infants had at least two measurements subsequent to their weight nadir, we assumed a linear weight gain between the last two recordings and extrapolated from this line. Thirteen infants (three breast fed, five formula fed, and five mixed fed) did not have two such measurements and were excluded at this point, leaving 924 infants. Twenty four babies had multiple data measurements into the second and third weeks of life but were not consistently showing any weight gain at this time. A quantitative estimate of the time to regain birth weight could not be provided for these babies, but the frequency of such cases was compared between the groups.

Eighty five babies had no recorded fall in weight (61 formula fed, 16 breast fed, eight mixed fed). These infants were included in the analysis; the timing of their first follow up weight ranged from 0.67 to 11.4 days (median 2.7 days in formula fed infants and 5.0 days in breast fed infants). It is important to include these infants, as the reasons for failure to record a fall in weight probably differed between the groups. For these infants, the minimum recorded weight ratio was taken as 1.0, the timing of the minimum weight was taken as the midpoint between birth and the time of the first follow up weight, and the time taken to regain birth weight was taken as the time of the first follow up weight.

\section{Statistical analysis}

Three variables were studied: the maximum recorded percentage weight loss, the timing of this weight loss, and the time taken to regain birth weight. The data distribution for each variable was studied using the Anderson Darling Normality test. This confirmed that the data were not normally distributed $(\mathrm{p}<0.001$ for each parameter). In view of the skewed distribution, the results are presented as medians and centiles, which were derived directly from the data by ordering and counting. The binomial distribution method was used to produce $95 \%$ confidence intervals for the centiles. Comparisons of the group medians between the breast and formula fed groups were carried out using a Mann-Whitney U test, and the Fisher exact test was used to compare proportions.

\section{RESULTS}

Table 2 gives the data for the three variables by feeding method. Weight loss for breast fed infants was substantially greater than for formula fed infants (median: 6.6\% v 3.5\%, $\mathrm{p}<0.0001$ ). The difference between the two groups persisted at the more extreme end of the spectrum of weight loss. The feeding method did not influence the timing of the weight nadir. Breast fed babies took longer to regain their birth weight than formula fed infants (median: $8.3 v 6.5$ days, $\mathrm{p}<0.0001)$. The smaller numbers in the mixed fed group led to wide confidence intervals. They followed a very similar pattern to the fully breast fed for all three parameters. Further details of the distribution of results in the breast and formula fed groups are provided by cumulative frequency graphs (fig l).

Twenty two of 417 babies in the breast fed group and two of 391 in the formula fed group had multiple weight measurements yet showed no evidence of any real weight gain in the second and third week of life. The 22 such breast fed infants had a median of six weight records (range four to eight), with the median time of the last record being 15 days (range 11-20), two thirds having weights recorded at or beyond 2 weeks of age. The data on these infants could not meaningfully contribute to the centile calculations for regaining birth weight. The typical pattern of growth in these infants was a variable degree of initial weight loss, followed by limited weight gain $(70-150 \mathrm{~g})$ and then a faltering of weight gain between 10 and 20 days. These babies represented $5.3 \%$ of the breast fed population $(95 \%$ confidence interval (CI) $3.1-7.4 \%$ ) compared with only $0.5 \%$ of the formula fed group $(\mathrm{p}<0.0001)$.

We studied the correlation between the three variables for each group and present these results along with the 95\% CI in table 3 . The correlations between the degree of the initial

\begin{tabular}{|c|c|c|c|c|c|c|}
\hline \multirow[b]{2}{*}{$\begin{array}{l}\text { Weight } \\
\text { record }\end{array}$} & \multicolumn{2}{|c|}{ Breast fed } & \multicolumn{2}{|c|}{ Formula fed } & \multicolumn{2}{|c|}{ Mixed fed } \\
\hline & $\begin{array}{l}\text { No of } \\
\text { records }\end{array}$ & Timing (days) & $\begin{array}{l}\text { No of } \\
\text { records }\end{array}$ & Timing (days) & $\begin{array}{l}\text { No of } \\
\text { records }\end{array}$ & Timing (days) \\
\hline 1st & 435 & $2.4(1.5-4.7)$ & 415 & $2.4(1.6-4.8)$ & 121 & $2.3(1.5-4.9)$ \\
\hline 2nd & 420 & $4.9(3.7-7.7)$ & 396 & $5.0(3.9-8.6)$ & 121 & $4.8(3.4-9.0)$ \\
\hline $3 r d$ & 380 & $7.3(6.0-10.7)$ & 333 & $7.6(6.1-10.5)$ & 112 & $7.3(5.4-10.9)$ \\
\hline 4 th & 269 & $9.8(8.2-13.3)$ & 203 & $9.9(8.9-12.9)$ & 80 & $9.8(7.67-13.9)$ \\
\hline 5 th & 124 & $12.8(9.9-15.2)$ & 62 & $13.2(10.0-14.9)$ & 25 & $11.9(9.4-14.8$ \\
\hline
\end{tabular}

Timing $=$ median (10th-90th centile range) . 
Table 2 Centile data for the timing and degree of initial weight loss and the timing of recovery of birth weight by feeding group

\begin{tabular}{|c|c|c|c|c|}
\hline & Median & 90th centile & 95th centile & 97.5th centile \\
\hline \multicolumn{5}{|l|}{ Weight loss (\%) } \\
\hline Breast $(n=420)$ & $6.6(6.3-6.9)$ & $10.6(10.3-11.2)$ & $11.8(11.2-12.9)$ & $12.8(12.1-13.7)$ \\
\hline Formula $(n=396)$ & $3.5(3.0-3.9)$ & $6.9(6.6-7.8)$ & $8.4(7.8-8.9)$ & $9.5(8.6-10.9)$ \\
\hline Mixed $(n=121)$ & $5.9(4.8-6.9)$ & $10.6(9.5-11.6)$ & $11.5(10.6-12.8)$ & \\
\hline \multicolumn{5}{|l|}{ Timing of loss (days) } \\
\hline Breast $(n=420)$ & $2.7(2.5-2.8)$ & $7.0(6.2-7.9)$ & $9.1(7.7-10.2)$ & $10.3(10.0-11.1)$ \\
\hline Formula $(n=396)$ & $2.7(2.5-2.9)$ & $6.2(5.5-6.8)$ & $7.1(6.7-9.2)$ & $9.3(7.9-9.9)$ \\
\hline Mixed $(n=121)$ & $2.5(2.2-2.8)$ & $6.5(4.9-10.0)$ & $9.3(6.5-12.0)$ & \\
\hline \multicolumn{5}{|c|}{ Regain birth weight (days) } \\
\hline Breast $(n=395)$ & $8.3(7.7-8.9)$ & 15.5 (14.5-17.3) & $18.7(16.7-20.8)$ & $21.0(19.8-24.0)$ \\
\hline Formula $(n=389)$ & $6.5(6.2-7.1)$ & $12.4(11.8-13.9)$ & $14.5(13.8-16.7)$ & $16.7(15.3-20.2)$ \\
\hline Mixed $(n=116)$ & $7.9(7.0-8.5)$ & $15.7(13.3-19.0)$ & $19.0(15.7-20.3)$ & \\
\hline
\end{tabular}

weight loss and its timing were weak. There were stronger associations between both the degree and the timing of the initial weight loss and the time to regain birth weight. These associations were consistently stronger for formula fed infants.
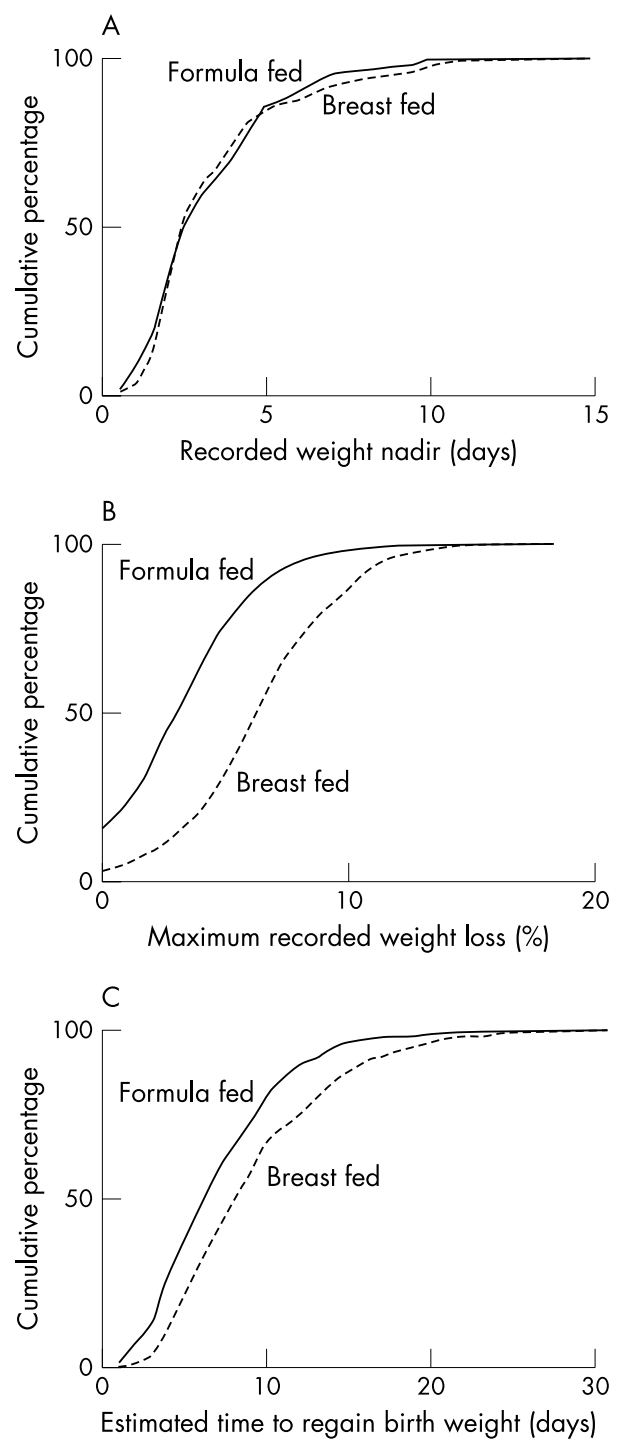

Figure 1 Cumulative distribution curves for the breast and formula fed groups for (A) weight nadir, (B) maximum weight loss, and (C) time to regain birth weight.
Electrolytes were measured in 48 babies who lost more than $10 \%$ of their birth weight; 37 were breast fed and 11 were mixed fed. Plasma sodium levels ranged from 138 to $159 \mathrm{mmol} / \mathrm{l}$. There is a significant correlation between weight loss and hypernatraemia ( $r=0.42,95 \%$ CI 0.15 to 0.63 ). Records of mild hypernatraemia (146-150 mmol/l) were found at all levels of weight loss. Some degree of hypernatraemia occurred in $73 \%$ of babies above the 95th centile for breast fed weight loss and was universal above the 97.5th centile for breast fed weight loss. Plasma sodium levels of $151-155 \mathrm{mmol} / \mathrm{l}$ were found in $80 \%$ of this last group.

\section{DISCUSSION}

Main findings

We have clearly shown that breast fed infants initially lose more weight and take longer to regain their birth weight than formula fed infants. However, within the limitations of the study, we did not identify any influence of feeding method on the timescale over which the initial weight loss occurred. We have reported upper centiles with confidence intervals, for both the degree and timing of initial weight loss and the time required to regain birth weight in newborn infants by feeding method. We hope that the information presented will aid clinical decision making in situations where there is concern about the adequacy of nutrition and growth in newborn infants.

\section{Weaknesses}

The main weakness of our study is that the intermittent weighing policy necessarily introduces an element of approximation. As an individual's maximum weight loss may be underestimated, the true population median and centiles for initial weight loss may be more extreme than reported. This is more likely to have happened for infants who started to gain weight soon after birth and had fewer low weights recorded. Thus it is likely to have influenced the centiles for lesser weight loss much more than the centiles for greater weight loss. It is the latter centiles that are more clinically useful.

The policy of intermittent weight recording may also fail to identify a real difference between two groups in the timing of the weight nadir. Infants who reached their nadir quickly would often do so on the first weight recording. As the median timing of the nadir was only 2.7 days, it is likely that this value is strongly influenced by the timing of the first weight (median 2.4 days). However, for the more extreme centiles, the nadir was recorded on the second, third, or even fourth measurement. The pragmatic approach taken to data collection meant that these records occurred over a time period that overlapped with that of adjacent records. The more extreme centiles are based on more data points. We 
Table 3 Correlation $(r)$ and 95\% confidence interval between parameters

\begin{tabular}{llll}
\hline Parameters correlated & Formula fed & Breast fed & Mixed fed \\
\hline $\begin{array}{l}\text { Timing and degree of initial } \\
\text { loss }\end{array}$ & $0.31(0.22$ to 0.40$)$ & $0.194(0.10$ to 0.23$)$ & $0.05(-0.13$ to 0.23$)$ \\
$\begin{array}{l}\text { Regaining birth weight and } \\
\text { timing of initial loss }\end{array}$ & $0.56(0.48$ to 0.62$)$ & $0.33(0.24$ to 0.42$)$ & $0.46(0.31$ to 0.60$)$ \\
$\begin{array}{l}\text { Regaining birth weight and } \\
\text { degree of initial loss }\end{array}$ & $0.66(0.60$ to 0.71$)$ & $0.44(0.36$ to 0.52$)$ & 0.53 (0.39 to 0.65$)$ \\
\hline
\end{tabular}

consider that they are less subject to influence by the timing of the weight measurements and are likely to be of greatest clinical value.

We derived the time taken to regain birth weight by extrapolating between two data points. Inevitably this involves approximation at the level of the individual, but the population effects are likely to be neutral. The faltering weight gain in 5.3\% of the breast fed group necessarily excluded some infants, who by definition were taking longer than their peers to regain birth weight, with the likely consequence of underestimation of the upper centiles for regaining birth weight in this group.

\section{Strengths}

Our results are derived from contemporary community based population data, with large numbers in each feeding group and a comprehensive inclusion policy that minimises the risk of sampling bias. The weight monitoring was carried on into the second and third week of life, and the data analysis and presentation concentrates on studying the upper extremes. In previous studies, breast fed infants were weighed on a daily basis. They have been based on smaller samples, a hospital population, and usually only the first 3-4 days of life..$^{3-7}$ These studies were not designed with the specific purpose of identifying population patterns of weight loss, and the data distribution has not been well reviewed. The reports concentrate on providing sample means and standard deviations, rather than paying specific attention to upper extremes. These studies report a mean weight loss of 5-6\%. Although our results are based on approximations, they are consistent with these reports. The complementary strength of our approach is that centiles estimated are based on a pattern of weight monitoring that can be practically duplicated within a real clinical scenario.

\section{Secondary findings}

We did not set out to comprehensively review the biochemical changes in an infant population. Our data on hypernatraemia are selective (all had at least 10\% weight loss but not all such infants were tested). It does show an association between the degree of weight loss and the degree of hypernatraemia. Mild hypernatraemia (146-150 mmol/l) was extremely common in the tested sample. However, this is probably not of clinical significance, as such degrees of plasma sodium increase have been documented in almost a third of breast fed infants, with all degrees of recorded weight loss. ${ }^{5}$ This should probably be considered to be a normal physiological process secondary to the inevitable period of limited fluid and energy intake during the first few days of life. More extreme degrees of hypernatraemia (> $150 \mathrm{mmol} /$ l) can occasionally occur with a relatively small weight loss, ${ }^{5}$ but the high frequency with which we observed such results in association with more extreme weight loss $(>97.5$ th centile) suggests an unusual degree of physiological stress.

The stronger correlation between the time taken to regain birth weight and the other two parameters for the formula fed infants suggests that there is more variability in the rate at which breast fed infants regain lost weight than in the formula fed population. Thus there may be some breast fed babies growing at a suboptimal rate who could benefit from additional assessment and support with feeding. This concept is further supported by the fact that faltering weight gain during the 2 nd or 3 rd week was seen in $5.3 \%$ of our breast fed babies.

\section{Context}

Once breast feeding is established, infants gain weight faster over the early months than current growth charts would suggest. ${ }^{7}$ However, feeding problems leading to early failure to thrive ${ }^{8}$ and severe dehydration ${ }^{9}$ in breast fed infants have been noted in the literature for well over 20 years, sometimes with high morbidity. ${ }^{10}$ Harding et al report one infant a month being readmitted with this problem in Bristol, and Oddie et $a^{2}$ calculate the minimum incidence of hospital readmission with this problem to be $0.2 \%$ in first time breast feeding mothers. Laing and Wong ${ }^{11}$ recently reviewed the literature on early hypernatraemic dehydration. They question whether the incidence of these cases is rising and call for a national audit. These groups all advocate monitoring the weight of infants until growth is established. However, past reports have not tried to specifically study the upper limits of the distribution, and acknowledgement of uncertainty as to "exactly how much is normal" weight $\operatorname{loss}^{2}$ legitimately raises questions as to the appropriateness of this response. Williams $^{12}$ questions the benefits of weight monitoring, implying that it may be harmful. He emphasises the need for training of health professionals (paediatricians included) in the management of breast feeding. Many others (in electronic responses to the paper of Oddie et al) reflected this view, considering that attention to details such as feeding technique, jaundice, and stool and urine frequency is the appropriate way to recognise such problems. However, it is clear from the widespread observation of newborns being readmitted with dehydration and/or failure to thrive that, although these signs are useful, relying on their recognition is not adequate on its own. Uninformed weighing may be harmful, but monitoring the weight of infants, informed by knowledge of population patterns, is the only objective method of identifying the mother and child in need of support. Late recognition of problems almost inevitably leads to the mother giving up breast feeding $\left(80 \%{ }^{1}\right.$ and $\left.87 \%{ }^{2}\right)$. The use of this weight centile data is not a substitute for training midwifery and paediatric staff in the clinical recognition and management of breast feeding problems. Rather, it is a tool that can supplement these clinical skills and provide a safety net, ensuring that those who could benefit most are offered additional support.

\section{Local application}

The breast feeding support strategies within our unit are consistent with the "Baby Friendly" initiative, and all medical and midwifery staff receive training in the support of breast feeding. Our data can inform weight monitoring of infants, and we now use it to identify mothers who require 
additional support. On the basis of the numbers of infants who showed faltering weight gain, we consider it likely that $5-10 \%$ of mothers would benefit from additional support. We follow a two tier approach to weight loss in the breast fed infant. We set a lower threshold for offering additional intensive personal breast feeding support by dedicated breast feeding support midwives. We aim to offer this extra support to around $10 \%$ of breast feeding mothers and do so if the breast fed infant loses $\geqslant 10 \%$ of the birth weight, does not start to gain weight by 9 days, or fails to regain the birth weight by 2 weeks of age. We set a higher threshold for medical referral and intervention. We allow up to $12.5 \%$ weight loss before considering biochemical testing and rehydration therapy and 3 weeks to regain birth weight before considering other nutritional supplementation. This two tiered approach ensures that most infants coming to medical attention have already received additional dedicated breast feeding support, with attention paid to issues such as positioning, feeding technique, and the use of hand expression with additional cup feeding. We also provide a medical review to exclude underlying organic illness in formula fed infants who lose $>10 \%$ of their birth weight or fail to regain their birth weight in 2 weeks.

\section{Conclusion}

Breast feeding is accepted to be the optimum method of newborn feeding. There is currently a nationwide effort to encourage increased breast feeding within a culture in which this has not been the norm. In such a situation, health professionals will have to deal with concerns about the success of lactation. It is important to recognise that problems can occur. Denial of such problems leads to failure to provide the necessary support to address the issues. Professionals can only deal with such situations confidently if they have adequate data on which to base their advice. Without these data, professional uncertainty may be communicated to the mother undermining her confidence in breast feeding. Monitoring the weight gain of newborns combined with a clear knowledge of population patterns allows additional support to be targeted at those who will benefit most. We believe that sensible use of our data makes this possible.

\section{ACKNOWLEDGEMENTS}

We would like to thank the midwifery staff for their diligent record keeping without which this study would not have been possible.

\section{Authors' affiliations}

P D Macdonald, S R M Ross, L Grant, Southern General Hospital Neonatal Paediatric Department, South Glasgow University Hospitals NHS Trust, Glasgow G51 4TF, Scotland, UK

D Young, Research \& Development Department, Yorkhill NHS Trust, Glasgow G3 8SJ

\section{REFERENCES}

1 Harding D, Cairns P, Gupta S, et al. Hypernatraemia: why bother weighing breast fed babies? Arch Dis Child Fetal Neonatal Ed 2001;85:F1 45.

2 Oddie S, Richmond S, Coultard M. Hypernatraemic dehydration and breast feeding: a population study. Arch Dis Child 2001;85:318-20.

3 Maisels MJ, Gifford K. Breast feeding, weight loss and jaundice. J Pediatr 1983;102:117-18.

4 Maisels MJ, Gifford K, Antle CE, et al. Jaundice in the healthy newborn infant: a new approach to an old problem. Pediatrics 1988;81:505-11.

5 Marchini G, Stock S. Thirst and vasopressin secretion counteract dehydration in newborn infants. J Pediatr 1997;130:736-9.

6 Marchini G, Fried G, Ostlund E, et al. Plasma leptin in infants: relations to birth weight and weight loss. Pediatrics 1998;101:429-32.

7 Dewey KG, Peerson JM, Brown KH, et al. Growth of breast fed infants deviates from current reference data: a pooled analysis of US, Canadian and European data sets. World Health Organisation Working Group on Infant Growth. Pediatrics 1995;96:495-503.

8 Evans TJ, Davies DP. Failure to thrive at the breast: an old problem revisited. Arch Dis Child 1977;52:974-5.

9 Clarke TA, Markarian M, Griswold W, et al. Hypernatremic dehydration resulting from inadequate breast feeding. Pediatrics 1979;63:931-2

10 Cooper WO, Atherton HD, Kahana M, et al. Increased incidence of severe breast feeding malnutrition and hypernatremia in a metropolitan area. Pediatrics 1995;96:957-60.

11 Laing IA, Wong CM. Hypernatraemia in the first few days: is the incidence rising? Arch Dis Child Fetal Neonatal Ed 2002;87:F158-62.

12 Williams AF. Weighing breast fed babies. Arch Dis Child Fetal Neonatal Ed 2002;86:F69. 